\title{
Post-menopausal vulvovaginal atrophy - an overview of the current treatment options
}

\author{
Jacek Krzysztof Szymański 1, 2, Bogumił Paweł Siekierski1, 2, Anna Kajdy 1, 2, Grzegorz Jakiel \\ ${ }^{1}$ St. Sophia Hospital in Warsaw, Poland \\ ${ }^{2}$ Department of Reproductive Health, Centre of Postgraduate Medical Education, Warsaw, Poland \\ ${ }^{3} 7^{\text {st }}$ Department of Obstetrics and Gynecology, Centre of Postgraduate Medical Education, Warsaw, Poland
}

\begin{abstract}
Sex hormone deficiency in post-menopausal women causes changes in the lower urinary tract. Vulvovaginal atrophy is a pathology resulting from those changes. VVA has a negative effect on the quality of life therefore prompting a search for new therapeutic options. The aim of this article is to summarize the current treatment modalities, both hormonal and non-hormonal for post-menopausal vaginal atrophy. Topical oestrogen therapy remains the "golden standard". Alternatives, although promising, require well-designed control studies.
\end{abstract}

Key words: vulvovaginal atrophy, genitourinary syndrome of menopause, hormonal vaginal therapy, non-hormonal vaginal therapy, laser vaginal atrophy

Ginekologia Polska 2018; 89, 1: 40-47

\section{INTRODUCTION}

The post-menopausal period comprises up to $1 / 3$ of a woman's life. Diminished ovarian function has an adverse effect on the quality of life. Hot flushes, night sweats, anxiety, mood swings, irritability and sleeping disorders often occur [1]. Sex hormone deficiency affects changes in many organs including the lower urinary tract. Oestrogens are the main regulators of physiological functions of the vagina. They influence maturation, adequate thickness and structure of the epithelium, proper vascular flow, quality and structure of the collagen, low vaginal $\mathrm{pH}$ and vaginal secretion. $40-60 \%$ of post-menopausal women suffer from atrophic changes of the vagina (VVA, vulvovaginal atrophy) [2]. These changes involve the thinning of the vaginal epithelium, a less prominent vaginal rugae, increased friability, irritation, burning and pruritus, contact bleeding and discomfort during sexual intercourse due to vaginal dryness. Moreover, decreased glycogen storage, that constitutes substrate for lactic acid production by Lactobacilli, leads to vaginal alkalization and the dysfunction of the physiological bacterial flora. The described constellation of signs and symptoms, accompanied by urgency, dysuria and recurrent urinary tract infections is labeled as the genitourinary syndrome of menopause (GSM) [3-5]. A multi-center, population study AGATA based on 913 women, mean age 59.3 , revealed the GSM prevalence in $79.1 \%$, occurring 1-6 years after menopause. In this observation, all women diagnosed with GSM suffered from vaginal dryness, 77.8\% reported dyspareunia, whereas burning, itching and dysuria occurred in $56.9 \%, 56.6 \%$ and $36.1 \%$ respectively [6]. These symptoms significantly decrease the quality of life, especially in the sexual sphere, which was confirmed by the REVIVE study, conducted on a group of 3046 post-menopausal women [7]. The CLOSER study showed that dyspareunia is the reason for the limitation or complete withdrawal from sexual life which substantially disturbs partner relations $[8,9]$, and often causes depression and decreased self-assessment [10]. Therefore, the aim of GSM/VVA therapy is the restoration of proper vaginal epithelium function, relief from the signs and symptoms caused by sex hormone deficiency and improvement in the quality of life with the special interest in sexual life. 


\section{AN OVERVIEW OF THE CURRENT TREATMENT OPTIONS Topical oestrogen therapy}

Topical vaginal oestrogens constitute the golden standard of VVA therapy. Oestrogens act on a molecular level by activation of the oestrogen receptors ER-alpha and ER-beta. ER-alpha receptors are localised in the uterus, ovaries, oviducts, mammary gland, hypothalamus, liver, fibroblasts and macrophages, while ERs-beta are found in the ovaries, central nervous, circulatory, immune, genitourinary and respiratory systems $[11,12]$. Both types of receptors are present in the vaginal wall, but their distribution is affected by the menstrual status. Chen et al [13] reported the expression of ER-alpha in the vagina of premenopausal and postmenopausal women, while ER-beta expression was found only in premenopausal women. These outcomes could be affected by a small number of the postmenopausal women (No-4). Gebhart et al [14] confirmed significant differences in the presence and expression of ER-alpha and ER-beta in the vaginal tissue between premenopausal and postmenopausal women. In the postmenopausal women, the expression of ER-beta in the vaginal wall was detected, but it was markedly reduced, regardless of oestrogen therapy. There are two ways of oestrogen receptor stimulation, classical and non-classical. The classical, genomic pathway, leads to receptor nuclear translocation induction and binding to EREs (oestrogen response elements). This has a direct effect on DNA which regulates transcription of target gens. The non-classical signalling concerns the membrane-associated receptor activation and induction of cytoplasmic pathways such as PI3K/Akt, ERK and p38. The stimulation of ER by the non-classical way causes rapid, immediate oestrogen effect. So far, the mechanism of choosing classical or non-classical pathway remains unknown. Oestrogen receptors also affect the activation of growth factors, such as EGFR, HER2/Neu and IGFR [15-19]. Sawczuk el al. [20] showed the relationship between both oestrogen receptor expression and the way of oestrogens administration. Local therapy caused higher ERs-alpha and ERs-beta density, compared to systemic treatment. The study revealed, that topical oestrogen therapy is more effective in stimulating epithelial vaginal receptors. The other authors conclude, that a therapeutic effect is limited only to the treatment period and abates shortly after stopping the therapy. Moreover, oestrogens influence only the epithelium, causing its thickening and stimulating secretion, with no effect on the extracellular matrix and neovascularization [21]. Recently animal models described oestrogens effect on collagen synthesis [22]. The 2017 Hormone Therapy Position Statement of The North American Menopause Society states that women with GSM symptoms only respond better to low-dose vaginal oestrogen therapy than systemic therapies. If sexual function is the main concern, women with menopause symptoms benefit from transdermal estrogen therapy (smaller effect on sex hormone-binding globulin and free testosterone levels) and from low-dose vaginal oestrogens. Moreover, in women without menopause symptoms systemic hormonal therapy does not improve sexual function [23]. One should keep in mind, that there are many limitations of the oestrogen therapy, such as hormone-dependent cancers, history of oestrogen-related thromboembolization and the adverse effects: nausea, vomiting, breast tenderness, vaginal bleeding but also the patient's anxiety of cancer and cultural or behavioural considerations $[24,25]$. There is no published data concerning the safety of longitudinal topical oestrogen therapy in certain women subpopulations [26]. Santen [27] reported the effect of vaginally administered oestradiol on $\mathrm{E}_{2}$ plasma level in a dose-dependent manner. Low-dose vaginal oestrogen (7.5-10 $\mu \mathrm{g}$ oestradiol) increased plasma oestradiol levels but not above the normal range of $\leq 20 \mathrm{pg} / \mathrm{mL}$. Surprisingly, these small increments were associated with systemic effects to decrease the bone resorption rate and to lower the serum levels of low density lipoprotein cholesterol. Intermediate doses ( $25 \mu \mathrm{g}$ oestradiol) resulted in plasma levels approaching or exceeding $20 \mathrm{pg} / \mathrm{mL}$, while the higher doses $(50-2000 \mu \mathrm{g})$ reached the premenopausal oestrogen serum levels. The amount of oestrogen systemically absorbed from the vagina is clinically relevant with regard to an increased risk of breast cancer, heart disease, venothrombotic and cerebrovascular events. Low-dose regimens should be recommended to the women with atrophic vaginitis as the initial therapy because they are as effective in the VVA treatment as higher dosages. On the other hand, there is also data concerning the lack of efficacy of local oestrogen therapy in $23-42 \%$ of treated women [7]. Furthermore, the recent Cochrane Database systematic review on local oestrogen for vaginal atrophy treatment concluded, that there was low-quality evidence for the improvement of the vaginal atrophy symptoms in postmenopausal women, when treated with local oestrogen agents compared to a placebo. The authors found also no evidence of a difference in efficacy between the various topical oestrogen regimens when compared with each other [28].

\section{LUBRICANTS AND LOCAL MOISTURISERS}

This is the most popular kind of local VVA therapy. There are several forms of vaginal lubricants and moisturisers, such as globules, creams and liquids. Their main component is hyaluronic acid, polysaccharide, which has the ability to conserve a large amount of water molecules, allowing proper levels of moistening of the extracellular matrix. Origoni et al [29] assessed the effectiveness of hyaluronic acid-based liquid regimen for vaginal use in 46 post-menopausal women 
during an 8-week therapy. The investigators used VAS (Visual Analogue Scale) to evaluate the score of the symptoms and VHI (Vaginal Health Index) by Gloria Bachmann [30]. VHI is an investigation tool clinically comprising of five vaginal parameters: elasticity, fluid volume, $\mathrm{pH}$, epithelial integrity and moisture. The degree of treatment satisfaction was assessed according to the Likert scale (very satisfied, satisfied, uncertain, dissatisfied, very dissatisfied). The study revealed a significant improvement in vaginal dryness, itching, burning and dyspareunia according to VAS and a 95\% degree of patents'satisfaction. Furthermore, an objective evaluation of vaginal mucosa according to $\mathrm{VHI}$ confirmed the subjective symptoms, showing statistically significant improvement of the mucosa quality. The obtained results are concurrent with the other authors' reports, evaluating the therapeutic effect of the hyaluronic acid in different pharmacological forms and treatment modes [31-33]. Some authors point out, that the therapeutic effect of hyaluronic acid is limited to the treatment period and does not involve collagen synthesis and cellular maturation [34, 35]. They emphasise solely the symptomatic aspect of this therapy, with no influence on vaginal epithelium physiology.

\section{SELECTIVE OESTROGEN RECEPTORS MODULATORS}

Ospemifene is the first oral non-steroid selective modulator of the oestrogen receptors with the affiliation to the vaginal mucosa. The effectiveness of $60 \mathrm{mg} /$ day ospemifene was assessed by Portman et al. [36] in a randomised, placebo-controlled, phase III trial on 314 women, who reported vaginal dryness as the most bothersome symptom of VVA. There were $33.8 \%$ responders in the treatment group compared to $7.1 \%$ of patients receiving a placebo. The evaluation included MI (Maturation Index), vaginal $\mathrm{pH}$ and the severity of the most bothersome symptom of vaginal dryness. Significant improvement in all assessed factors was seen in the treatment group during a minimum 10-week therapy. Adverse effects were rare although one case of deep vein thrombosis was noted and three patients discontinued therapy due to the worsening of hot flushes. The three patients in the ospemifene group and one in the placebo group had an endometrial thickness $>5 \mathrm{~mm}$ after 12 weeks of the therapy with no pathological changes in the histology samples. The other randomised III phase trial evaluated effectiveness of ospemifene in vaginal dryness and dyspareunia treatment [37]. Double blind, 12-week study was conducted on 919 women, based on FSFI (Female Sexual Function Index) assessing six domains: desire, arousal and lubrication, orgasm, satisfaction and pain. The serum levels of oestradiol, FSH, LH, SHBG and testosterone were measured during screening and on the final visit. In the treatment group, general improvement in FSFI was noticed, and was statistically significant for dyspareunia, with no significant improvement in vaginal dryness. A slight decrease of FSH and LH level was observed, while still in the normal limits for post-menopausal women. No changes in oestradiol level were noticed, the mean SHBG level increased, but remained within normal range. The total testosterone levels increased unremarkably in the ospemifene group, but the mean free testosterone level remained unchanged. The obtained data suggests, that the improvement confirmed by FSFI is not related to serum hormone changes but demonstrate the beneficial effect of ospemifene on vaginal mucosa. Other authors also described the effectiveness of ospemifene compared to a placebo as well as the safety of this therapy [38-42]. A recent review on ospemifene in the VVA treatment reported also that an oestrogenic effect of ospemifene on the bone is comparable to the bone-restoring activity of raloxifene. The authors highlighted an oestrogen antagonistic effect of ospemifene in animal models of breast cancer as well as no adverse effects on the breast in clinical trials with postmenopausal women at 52-weeks follow-up. The review did not report any case of endometrial carcinoma. The studies demonstrated no significant risk of endometrial hyperplasia associated with the use of ospemifene, however the safety of treatment longer than 1 year is uncertain. No adverse effects of ospemifene on lipid profile were observed. The ospemifene therapy increases the risk of vein thromboembolism (1.45/1000 women in the ospemifene group and $1.04 / 1000$ women in the placebo group), thus this treatment is contraindicated in women with thromboembolic disease or a history of these condition. Ospemifene has met the requirements of FDA (The Food and Drug Administration) for primary efficacy in vaginal atrophy and has recently been approved by the European Medicines Agency (EMA) to treat moderate-to-severe VVA in postmenopausal women who are not subject to local vaginal estrogen therapy $[43,44]$. Lasofoxifene is the next drug in this group. Its affiliation to both types of oestrogen receptors improves vaginal secretion and decreases $\mathrm{pH}$. Furthermore, similar to other SERM, like raloxifene, lasofoxifene exerts positive effects on other menopausal afflictions, such as osteoporosis and coronary disease $[5,45]$.

Phytoestrogens are another alternative to oestrogens. QiBaoMeiRan (QBMR) is undergoing clinical trials, which is a herbal regimen originating from traditional Chinese medicine. This is a mixture of the roots of: Polygoni Multiflori, Angelicae Sinensis, Achyrantis Bidentatae, the seeds of Cuscutae and the fruits of Lycii, Poria and Psoraleae. The mechanism of action is an agonistic/antagonistic impact on oestrogen receptors alpha and beta, resulting in expression ERs-beta, which makes the QBMR act like SERM. The studies on animal models revealed a proliferative effect on the vaginal epithelium without such an impact on 
endometrial tissue. Oestrogen acts through ERs stimulation including EREs pathway. QBMR therapy increases serum oestradiol levels with a concurrent decrease of FSH and LH levels. This data suggests that the effect of QBMR may be mediated through the hypothalamus - pituitary - ovary axis, which stimulates the biosynthesis of the oestrogens in the ovary [46]. Although the animal studies seem promising, the future of QBMR in the treatment of VVA in postmenopausal women remains uncertain.

\section{TOPICAL USE OF THE GROWTH FACTORS}

KGF (Keratinocyte Growth Factor) cream could be a competitive VVA therapy to local oestrogen administration. KGF is a member of the fibroblasts growth factors family (FGFs). It demonstrates a cytoprotective and regenerative activity, and deals with the maintenance of the integrity and functionality of epithelial tissues. KGF acts by binding the receptor tyrosine kinase and probable activation of an alternative signalling pathway that is able to stimulate ER-alpha through the phosphorylation of Ser118, as well as oestradiol. KGF administration in the developing vagina of mice results in the proliferation of the vaginal epithelium, suggesting a potential relationship between oestrogen treatment and activation of KGF pathway [47, 48]. Ceccarelli et al. [49] compared, an animal model in vivo and local vaginal KGF and $E_{2}$ therapy, showing a positive impact of KGF on vaginal epithelium proliferation, without systemic effects. In contrast to oestrogens, KGF does not affect the genomic pathway EREs, moreover it can prevent the activation of this pathway by retaining ER-alpha at the plasma membrane, thus avoiding its nuclear translocation. Besides, oestrogen-independent KGF activity, it acts on non-oestrogen-sensitive, ER-alpha negative cells. These observations suggest the existence of an overlapping between oestrogen and KGF signalling. Topical administration of KGF causes the recovery of vaginal atrophy in ovariectomized mice, thus provides a rationale for its pre-clinical development in humans [49]. Further studies are required to assess the efficiency of KGF in the VVA therapy in postmenopausal women.

\section{LASER AND RADIO-FREQUENCY DEVICES IN VVA TREATMENT}

Positive experiences with the energy transmitting devices in stimulating reparative and regenerative processes in tissues, prompted an interest in applying these possibilities in GSM therapy.

\section{RADIO-FREQUENCY DEVICES}

Radio-frequency (RF) devices emit high frequency wavelengths, which meet with the tissue impedance, resulting in a local temperature increase. The temperature at $40-45^{\circ} \mathrm{C}$ induce synthesis of collagen by fibroblasts. These devices are used for nonsurgical vulvovaginal correction procedures, as well as for the improvement of the elasticity, tension and rejuvenation of the vagina. The treatment sessions are painless, well tolerable, with the duration spans of 15-30 min. and the patients can resume their usual activities the same day. In 2015, the results of treatment of vulvovaginal laxity, sexual dysfunction and mild to moderate stress urinary incontinence with RF-based device, were published [50]. This prospective study involved 23 women including 6 premenopausal and 5 menopausal. The sessions lasted approximately 30 minutes with a working temperature range $40-45^{\circ} \mathrm{C}$. The treatment protocol consisted of three sessions with an interval of 4-6 weeks. There were no restrictions in normal patients' activity, including sexual intercourse immediately after each treatment. Significant improvement in vaginal tightening and laxity, as well as sexual satisfaction and decrease in urinary incontinence were noted. Treatments were well tolerated and no adverse effects were reported. Similar results received by Vicariotto et al. [51] during a twelve-months observation of 25 post-menopausal women with vaginal laxity and VVA/GSM-related symptoms treated by a low-energy dynamic quadripolar radio-frequency device. A therapeutic effect was measured according to VLQ (Vaginal Laxity Questionnaire), PISQ-12 (Pelvic Organ Prolapse/Urinary Incontinence Sexual Questionnaire), SSQ (Sexual Satisfaction Questionnaire) in the vaginal laxity arm and VAS (Visual Analogue Scale) for the assessment of sexual satisfaction in the vaginal atrophy arm. Rapid and long-lasting improvement in both groups in all evaluated parameters was noted.

\section{LASER DEVICES IN VVA THERAPY}

There are two types of lasers used in the vaginal revitalisation procedures: non ablative Erbium: YAG laser and ablative, $\mathrm{CO}_{2}$ fractional laser. Tissue effects ranges from a destructive one to thermal only, depending on the laser energy delivered and the delivery time.

\section{LASER ERBIUM: YAG}

Erbium:YAG is non-ablative laser, generating 2.940-nm wavelength that emits energy in the mid-infrared invisible light spectrum, with high absorption in water. One of these devices, working in "smooth" mode, produces a fast sequence of low-fluency laser pulses inside an overall super-long pulse allowing the heat to dissipate approximately $200 \mu \mathrm{m}$ into the mucosa, where the cumulative thermal effect stimulates collagen remodelling and new collagen synthesis. Gaspar et al. [21] compared the effectiveness of laser Erbium: YAG with topical estrogen in VVA treatment on a group of 50 post-menopausal women. The laser treatment arm first received a two-week pre-treatment with $0.5 \mathrm{mg}$ oestriol ovules to improve hydro-retention of the 
tissue. Water is a chromophore for the wavelength emitted by Erbium: YAG laser (the substance that absorbs photon energy, dispersing it as thermal energy). Then a total of three treatments, once every three weeks, were performed. The control group received an 8-week treatment of $0.5 \mathrm{mg}$ oestriol ovules, administered daily during the first week, three times a week in weeks 2-4, then twice a week in weeks 4-8. In both groups, a statistically significant reduction of vaginal dryness, leukorrhea, irritation and dyspareunia were noted, whereas in the laser arm the relief of symptoms was more prominent and remained at the 12-18 months follow-up. In the control group the therapeutic effect diminished at the 12-month follow up and at 18-month of observation some were even significantly worse than before treatment. After 12 months, in both groups MV (Maturity Value) of the epithelium improved, but in the laser arm improvement was much higher compared to control (52.2 and 25 points, respectively). Maturity Value determines the degree of the epithelium oestrogenisation. Oestrogenic stimulation is assessed by values of: 0-49 is an absence or low, 50-64 is medium and 65-100 is high. The $\mathrm{pH}$ value showed a statistically significant decrease in both groups, although the decrease in the oestriol group was not spectacular and had diminished at the 12-month follow-up. A histological examination in the laser arm revealed an increase of the epithelial thickness accompanied by parakeratosis, vasodilatation, improvement in vascularisation and increased collagenesis in the extracellular matrix. The side effects in both groups were transient and minimal. In the laser group, $4 \%$ of patients reported mild-to-moderate pain and slight transient mucosal edema related to sensation of warmth. In the control group $12 \%$ experienced abdominal pain, $8 \%$ vaginal spotting and $4 \%$ mastodynia. Similar results were obtained by Gambacciani et al. [52], while comparing Erbium: YAG laser treatment to oestrogen topical gel in 70 post-menopausal women with VVA. Significant reduction of the vaginal dryness, dyspareunia and urinary incontinence were noticed after a 6 month follow up. The VELAS (Vaginal Erbium Laser Academy Study) - an international multicenter observational study on the group of 1500 post-menopausal women is currently underway to evaluate the efficacy and safety of Erbium:YAG laser for the treatment of GSM and urinary incontinence [53].

\section{FRACTIONAL $\mathrm{CO}_{2}$ LASER IN THE TREATMENT OF VVA}

$\mathrm{CO}_{2}$ lasers are the members of the ablative lasers family. They emit wavelengths of $10.600 \mathrm{~nm}$ that cause thermal micro-damage of only a small fraction of the epithelium, leaving the rest of the intervening area unaltered. Minor affiliation to water of the $\mathrm{CO}_{2}$ laser as compared to Erbium: YAG laser, and ablation of the tissue allows the delivery of energy deeply into epithelium. Producers created various shapes of energy pulses. One of them is designed especially for the treatment of the vaginal mucosa. The shape of this pulse is divided into two distinct areas. The aim of the first impulse, set at $60 \mu \mathrm{s}$, is to perform a vaporisation of the water-deficient and poor heat conducting epithelium. This impulse opens the way for the second one, lasting from 100 to $2000 \mu \mathrm{s}$, with a peak power between 3 and $40 \mathrm{~W}$, that transfer a suitable thermal energy to the connective tissue in order to stimulate the reparative process. The damage of the epithelium constitutes of evenly spread points, $200 \mu \mathrm{m}$ each, covering only $6 \%$ of the tissue. Pitsouni et al. [54] evaluated the efficacy of $\mathrm{CO}_{2}$ laser - SmartXide ${ }^{2} \mathrm{~V}^{2} \mathrm{LR}$, in the treatment of GSM on the group of 53 post-menopausal women. Signs and symptoms, such as dyspareunia, vaginal dryness, itching, burning and dysuria, urgency, frequency and general sexual satisfaction were analysed using Visual Analogue Scale. The efficiency of laser therapy of LUTS (Lower Urinary Tract Symptoms) was assessed using ICIQ (International Consultation on Incontinence Questionnaires). The patients also fulfilled KHQ (King's Health Questionnaire), UDI-6 (Urogenital Distress Inventory) and PGI-I (Patients Global Impression of Improvement), evaluating general satisfaction at the end of the study. Additionally, vaginal $\mathrm{pH}, \mathrm{MI}$ and MV were analysed. Clinical assessment of the vaginal mucosa was evaluated using VHI score. All questionnaires revealed a significant reduction of VVA and LUT symptoms at the 12-week follow up. A significant reduction of symptoms or total remission reported $85 \%$ of patients. 27/30 women with a history of frequency above 9 micturition/day experienced significant improvement. The frequency of sexual intercourse/month increased from a mean 1.6 to 4.1. An increase of MV and VHI is a subject of special interest. At the 12-week follow up, $57 \%$ and $89 \%$ of the participants overpassed the threshold MV $>49 \%$ and $89 \% \mathrm{VHI}>15$, respectively, with none presenting such results at the baseline. This data indicates the oestrogen-like effect of $\mathrm{CO}_{2}$ laser on vaginal mucosa, regardless of the time of menopause. This conclusion was reflected in the Vaginal Health Index. Vaginal atrophy remission was observed in 47 participants. Furthermore, PGI-I revealed $90 \%$ high satisfaction of the treatment. The authors pointed out, that it was difficult to identify factors predicting which women could benefit the best from $\mathrm{CO}_{2}$ - laser therapy. Similar results were obtained by Siliquini et al. [55] in a 15-month follow-up of 87 post-menopausal women and Behnia-Willison et al. [56] in a 12-month observation of 102 women with GSM symptoms. The participants were treated with the fractional $\mathrm{CO}_{2}$ laser - SmartXide ${ }^{2} \mathrm{~V}^{2} \mathrm{LR}$, across a series of sessions performed at intervals of six or more weeks. The data was gathered using the Australian Pelvic Floor Questionnaire, which integrated bladder, bowel, sexual function, pelvic 
organ prolapse, severity, bothersome and condition-specific quality of life. The reduction of GSM symptoms in $84 \%$ of patients was noted. $39.5 \%$ of the participants reported improvement of sexual function at the 12-24 month follow up. Moreover, significant improvement was observed as far as pelvic organ prolapse, vaginal secretion, bladder function and urgency were concerned. Complications were rare with slight clinical significance: three women experienced post-coital urinary tract infections, two reported vaginal discharge, three women experienced lower pelvic pain, one had genital herpes and finally two women presented postmenopausal bleeding (benign in endometrial biopsies). The described clinical observations were confirmed by Zerbinati et al. [57] in a histological evaluation of the vaginal biopsies using light and electron microscopy. The study was conducted on a group of 50 post-menopausal women presenting GSM symptoms with $\mathrm{CO}_{2}$ laser - SmartXide ${ }^{2} V^{2}$ LR. Vaginal biopsy was performed at the baseline, as well as after 30 and 60 days of treatment. The investigation revealed spectacular changes in the vaginal mucosa. A thicker epithelium with a basal layer of proliferating cells and a significant storage of glycogen in the cells forming the intermediate and superficial layer indicating a restored mechanism oriented to the synthesis of glycogen was noted. Additionally, a high degree of exfoliation with superficial cells was observed. A light microscopy examination revealed a rich content of blood vessels in the connective tissue penetrating the newly-formed papillae, supporting the activity of fibroblasts. An ultrastructural investigation of the cells showed extended rough endoplasmic reticulum and vesicles for the synthesis and storage of procollagen molecules as well as a well-developed Golgi apparatus with associated vesicles for the glycosylation of proteins for the production of components of the extracellular matrix. To sum up, $\mathrm{CO}_{2}$-fractional laser therapy causes vaginal rejuvenation with structural changes specific for childbearing age with no need for hormonal treatment. A very interesting meta-analysis was performed by Pitsouni et al. [58]. The authors, based on 14 trials including laser treatment of 542 women, demonstrated reduction of all GSM symptoms. However, due to heterogeneity of treated populations, differences in diagnoses and different treatment protocols, the quality of evidence was "low" or "very low". In the authors' conclusion, although laser therapy seems promising, currently, evidence-based change in clinical practice for the management of GSM cannot be proposed. Well-designed controlled studies with standardised laser settings and therapeutic protocols are necessary.

There currently are several possibilities of GSM treatment available. Topical oestrogen treatment constitutes "the golden standard", based on the pathophysiology of this affliction. A well conducted treatment should be personalised to achieve an optimal effect with reduction of the complications. Hormonal treatment is not suitable for every patient. Nowadays, non-hormonal treatment options, such as local creams and gels as well as RF-based and laser devices are performed. Some of these therapies produce more spectacular and long-lasting results compared to hormonal treatment and the hormone-like effect in the vaginal mucosa obtained by physical factors is also fascinating. It seems advisable to conduct more studies concerning vaginal physiology and biostructure, in order to optimise GSM therapy and improve the quality of life of the post-menopausal women.

\section{REFERENCES}

1. Abdi F, Darooneh T, Ghorbani M, et al. Transdermal hormone replacement therapy with nanostructured medicines. Ginekol Pol. 2017; 88(2): 103-108, doi: 10.5603/GP.a2017.0018, indexed in Pubmed: 28326520.

2. Palacios S. Managing urogenital atrophy. Maturitas. 2009;63(4):315-318, doi: 10.1016/j.maturitas.2009.04.009, indexed in Pubmed: 19493638.

3. Portman DJ, Gass MLS. Vulvovaginal Atrophy Terminology Consensus Conference Panel. Genitourinary syndrome of menopause: new terminology for vulvovaginal atrophy from the International Society for the Study of Women's Sexual Health and the North American Menopause Society. J Sex Med. 2014; 11(12): 2865-2872, doi: 10.1111/jsm.12686, indexed in Pubmed: 25155380.

4. Farrell AE. Genitourinary syndrome of menopause. Aust Fam Physician. 2017; 46: 481.

5. Gandhi J, Chen A, Dagur G, et al. Genitourinary syndrome of menopause: an overview of clinical manifestations, pathophysiology, etiology, evaluation, and management. Am J Obstet Gynecol. 2016; 215(6): 704-711, doi: 10.1016/j.ajog.2016.07.045, indexed in Pubmed: 27472999.

6. Palma F, Volpe A, Villa $P$, et al. Vaginal atrophy of women in postmenopause. Results from a multicentric observational study: The AGATA study. Maturitas. 2016; 83: 40-44, doi: 10.1016/j.maturitas.2015.09.001.

7. Kingsberg $S A$, Wysocki $S$, Magnus $L$, et al. Vulvar and vaginal atrophy in postmenopausal women: findings from the REVIVE (REal Women's Vlews of Treatment Options for Menopausal Vaginal ChangEs) survey. J Sex Med. 2013; 10(7): 1790-1799, doi: 10.1111/jsm.12190, indexed in Pubmed: 23679050.

8. Simon JA, Nappi RE, Kingsberg SA, et al. Clarifying Vaginal Atrophy's Impact on Sex and Relationships (CLOSER) survey: emotional and physical impact of vaginal discomfort on North American postmenopausal women and their partners. Menopause. 2014; 21(2): 137-142, doi: 10.1097/GME.0b013e318295236f, indexed in Pubmed: 23736862.

9. Thomas $\mathrm{HM}$, Bryce $\mathrm{CL}$, Ness RB, et al. Dyspareunia is associated with decreased frequency of intercourse in the menopausal transition. Menopause. 2011; 18(2): 152-157, doi: 10.1097/gme.0b013e3181eeb774, indexed in Pubmed: 20962696.

10. Ambler DR, Bieber EJ, Diamond MP. Sexual function in elderly women: a review of current literature. Rev Obstet Gynecol. 2012; 5(1): 16-27, indexed in Pubmed: 22582123.

11. Brodowska A, Laszczynska M, Starczewski A, et al. The localization of estrogen receptor alpha and its function in ovaries of postmenopausal women. Folia Histochem Cytobiol. 2007; 45: 325.

12. Taylor AH, Al-Azzawi F. Immunolocalisation of oestrogen receptor beta in human tissues. J Mol Endocrinol. 2000; 24(1): 145-155, indexed in Pubmed: 10657006.

13. Chen GD, Oliver RH, Leung BS, et al. Estrogen receptor alpha and beta expression in the vaginal walls and uterosacral ligaments of premenopausal and postmenopausal women. Fertil Steril. 1999; 71(6): 1099-1102, indexed in Pubmed: 10360917.

14. Gebhart JB, Rickard DJ, Barrett TJ, et al. Expression of estrogen receptor isoforms alpha and beta messenger RNA in vaginal tissue of premenopausal and postmenopausal women. Am J Obstet Gynecol. 2001; 185(6): 1325-30; discussion 1330, doi: 10.1067/mob.2001.119627, indexed in Pubmed: 11744904

15. Fagan DH, Yee D. Crosstalk between IGF1R and estrogen receptor signaling in breast cancer. J Mammary Gland Biol Neoplasia. 2008; 13(4): 423-429, doi: 10.1007/s10911-008-9098-0, indexed in Pubmed: 19003523. 
16. Fagan DH, Uselman RR, Sachdev D, et al. Acquired resistance to tamoxifen is associated with loss of the type I insulin-like growth factor receptor: implications for breast cancer treatment. Cancer Res. 2012; 72(13): 3372-3380, doi: 10.1158/0008-5472.CAN-12-0684, indexed in Pubmed: 22573715.

17. Fan P, Wang J, Santen RJ, et al. Long-term treatment with tamoxifen facilitates translocation of estrogen receptor alpha out of the nucleus and enhances its interaction with EGFR in MCF-7 breast cancer cells. Cancer Res. 2007; 67(3): 1352-1360, doi: 10.1158/0008-5472.CAN-06-1020, indexed in Pubmed: 17283173 .

18. Fan P, Maximov PY, Curpan RF, et al. The molecular, cellular and clinical consequences of targeting the estrogen receptor following estrogen deprivation therapy. Mol Cell Endocrinol. 2015; 418 Pt 3: 245-263, doi: 10.1016/j.mce.2015.06.004, indexed in Pubmed: 26052034.

19. Shou J, Massarweh S, Osborne CK, et al. Mechanisms of tamoxifen resistance: increased estrogen receptor-HER2/neu cross-talk in ER/HER2-positive breast cancer. J Natl Cancer Inst. 2004; 96(12): 926-935, indexed in Pubmed: 15199112

20. Sawczuk B, Gołębiewska M, Mazurek A, et al. Immunohistochemical evaluation of oestrogen receptors $\alpha$ and $\beta$ in epithelium of the vaginal mucous membrane in women after oestrogen therapy. Prz Menopauzalny. 2017; 16(1): 12-18, doi: 10.5114/pm.2017.66178, indexed in Pubmed: 28546802

21. Gaspar A, Brandi H, Gomez V, et al. Efficacy of Erbium:YAG laser treatment compared to topical estriol treatment for symptoms of genitourinary syndrome of menopause. Lasers Surg Med. 2017; 49(2): 160-168, doi: 10.1002/Ism.22569, indexed in Pubmed: 27546524.

22. Montoya TI, Maldonado PA, Acevedo JF, et al. Effect of vaginal or systemic estrogen on dynamics of collagen assembly in the rat vaginal wall. Biol Reprod. 2015; 92(2): 43, doi: 10.1095/biolreprod.114.118638, indexed in Pubmed: 25537371.

23. Pinkerton JV, et al. Sánchez Agirre F, Blake JThe 2017 hormone therapy position statement of The North American Society. Menopause. 2017; 24:278.

24. Bachmann GA, Nevadunsky NS, et al. Diagnosis and treatment of atrophic vaginitis. Am Fam Physician 2000, 61, 3090.

25. Sousa MS, Peate $M$, Jarvis $S$, et al. A clinical guide to the management of genitourinary symptoms in breast cancer survivors on endocrine therapy. Ther Adv Med Oncol. 2017; 9(4): 269-285, doi: 10.1177/1758834016687260, indexed in Pubmed: 28491147.

26. Kokot-Kierepa M, Bartuzi A, Kulik-Rechberger B, et al. Lokalna terapia estrogenowa - implikacje kliniczne- 2012 update. Ginekol Pol. 2012; 83: 772 .

27. Santen RJ. Vaginal administration of estradiol: effects of dose, preparation and timing on plasma estradiol levels. Climacteric. 2015; 18(2): 121-134, doi: 10.3109/13697137.2014.947254, indexed in Pubmed: 25327484.

28. Lethaby A, Ayeleke RO, Roberts H. Local oestrogen for vaginal atrophy in postmenopausal women. Cochrane Database Syst Rev. 2016(8): CD001500, doi: 10.1002/14651858.CD001500.pub3, indexed in Pubmed: 27577677

29. Origoni M, Cimmino C, Carminati G, et al. Postmenopausal vulvovaginal atrophy (VVA) is positively improved by topical hyaluronic acid application. A prospective, observational study. Eur Rev Med Pharmacol Sci. 2016; 20(20): 4190-4195, indexed in Pubmed: 27831658.

30. Bachmann G. Urogenital ageing: an old problem newly recognized. Maturitas. 1995; 22 Suppl: S1-S5, indexed in Pubmed: 8775770.

31. Jokar A, Davari T, Asadi N, et al. Comparison of the Hyaluronic Acid Vaginal Cream and Conjugated Estrogen Used in Treatment of Vaginal Atrophy of Menopause Women: A Randomized Controlled Clinical Trial. Int J Community Based Nurs Midwifery. 2016; 4(1): 69-78, indexed in Pubmed: 26793732

32. Le Donne M, Caruso C, Mancuso A, et al. The effect of vaginally administered genistein in comparison with hyaluronic acid on atrophic epithelium in postmenopause. Arch Gynecol Obstet. 2011; 283(6): 1319-1323, doi: 10.1007/s00404-010-1545-7, indexed in Pubmed: 20577750.

33. Tersigni C, Di Simone N, Tempestilli E, et al. Non-hormonal treatment of vulvo-vaginal atrophy-related symptoms in post-menopausal women. J Obstet Gynaecol. 2015; 35(8): 835-838, doi: 10.3109/01443615.2015.1014326, indexed in Pubmed: 25968636.

34. Sturdee DW, Panay N. Recommendations for the management of postmenopausal vaginal atrophy. Climacteric. 2010; 13(6): 509-522, doi: 10.3109/13697137.2010.522875.

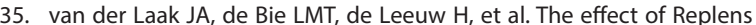
on vaginal cytology in the treatment of postmenopausal atrophy: cytomorphology versus computerised cytometry. J Clin Pathol. 2002; 55(6): 446-451, indexed in Pubmed: 12037029.

36. Portman D, Palacios S, Nappi RE, et al. Ospemifene, a non-oestrogen selective oestrogen receptor modulator for the treatment of vaginal dryness associated with postmenopausal vulvar and vaginal atrophy: a randomised, placebo-controlled, phase III trial. Maturitas. 2014; 78(2): 91-98, doi: 10.1016/j.maturitas.2014.02.015, indexed in Pubmed: 24679891.

37. Constantine G, Graham S, Portman DJ, et al. Female sexual function improved with ospemifene in postmenopausal women with vulvar and vaginal atrophy: results of a randomized, placebo-controlled trial. Climacteric. 2015; 18(2): 226-232, doi: 10.3109/13697137.2014.954996, indexed in Pubmed: 25252699.

38. Bachmann GA, Komi JO. Ospemifene Study Group. Ospemifene effectively treats vulvovaginal atrophy in postmenopausal women: results from a pivotal phase 3 study. Menopause. 2010; 17(3): 480-486, doi: 10.1097/gme.0b013e3181c1ac01, indexed in Pubmed: 20032798.

39. Simon J, Lin V, Radovich C, et al. One-year long-term safety extension study of ospemifene for the treatment of vulvar and vaginal atrophy in postmenopausal women with a uterus. Menopause:The Journal of The North American Menopause Society. 2012: 1, doi: 10.1097/gme.0b013e31826d36ba.

40. Simon J, Portman D, Mabey RG, et al. Ospemifene Study Group. Long-term safety of ospemifene (52-week extension) in the treatment of vulvar and vaginal atrophy in hysterectomized postmenopausal women. Maturitas. 2014; 77(3): 274-281, doi: 10.1016/j.maturitas.2013.12.005, indexed in Pubmed: 24411556.

41. Portman D, Bachmann G, Simon J. Ospemifene, a novel selective estrogen receptor modulator for treating dyspareunia associated with postmenopausal vulvar and vaginal atrophy. Menopause. 2013; 20(6): 623-630, doi: 10.1097/gme.0b013e318279ba64.

42. Alvisi S, Baldassarre M, Martelli V, et al. Effects of ospemifene on vaginal epithelium of post-menopausal women. Gynecological Endocrinology. 2017; 33(12): 946-950, doi: 10.1080/09513590.2017.1332589.

43. Shin JJ, Kim SKi, Lee JR, et al. Ospemifene: A Novel Option for the Treatment of Vulvovaginal Atrophy. J Menopausal Med. 2017; 23(2): 79-84, doi: 10.6118/jmm.2017.23.2.79, indexed in Pubmed: 28951854.

44. European Medicines Agency Summary of opinion Senshio (ospemifene) http://www ema europa eu/docs/en_GB/document_library/Summary of opinion - Initial_authorisation/human/002780/WC500177633 pdf (access: 2017.12.11)

45. Karwowski W, Lekesiz K, Koc-Żórawska E, et al. Effects of 17ß-estradioland raloxifene on endothelial OPG and RANKL secretion. Ginekol Pol. 2017; 88(4): 167-173, doi: 10.5603/GP.a2017.0033, indexed in Pubmed: 28509316.

46. Xu Y, Ma Xp, An Jn, et al. Short-time QiBaoMeiRan Formula Treatment Exerts Estrogenic Activities without Side Effects on Reproductive Tissues in Immature Mice. Sci Rep. 2015; 5: 17436, doi: 10.1038/srep17436, indexed in Pubmed: 26644197.

47. Masui F, Matsuda M, Mori T. Involvement of keratinocyte growth factor (KGF)-KGF receptor signaling in developmental estrogenization syndrome of mouse vagina. Cell Tissue Res. 2004; 318(3): 591-598, doi: 10.1007/s00441-004-0980-9, indexed in Pubmed: 15480797.

48. Hom YK, Young $P$, Thomson AA, et al. Keratinocyte growth factor injected into female mouse neonates stimulates uterine and vaginal epithelial growth. Endocrinology. 1998; 139(9): 3772-3779, doi: 10.1210/endo.139.9.6182, indexed in Pubmed: 9724029.

49. Ceccarelli S, D'Amici S, Vescarelli E, et al. Topical KGF treatment as a therapeutic strategy for vaginal atrophy in a model of ovariectomized mice. J Cell Mol Med. 2014; 18(9): 1895-1907, doi: 10.1111/jcmm.12334, indexed in Pubmed: 25088572.

50. Karcher C, Sadick N. Vaginal rejuvenation using energy-based devices. Int J Womens Dermatol. 2016; 2(3): 85-88, doi: 10.1016/j.jiwd.2016.05.003, indexed in Pubmed: 28492016.

51. Vicariotto F, DE Seta F, Faoro V, et al. Dynamic quadripolar radiofrequency treatment of vaginal laxity/menopausal vulvo-vaginal atrophy: 12-month efficacy and safety. Minerva Ginecol. 2017; 69(4): 342-349, doi: 10.23736/S0026-4784.17.04072-2, indexed in Pubmed: 28608667.

52. Gambacciani M, Levancini $M$, Cervigni M. Vaginal erbium laser: the second-generation thermotherapy for the genitourinary syndrome of menopause. Climacteric. 2015; 18(5): 757-763, doi: 10.3109/13697137.2015.1045485.

53. Gambacciani M, Torelli MG, Martella L, et al. Rationale and design for the Vaginal Erbium Laser Academy Study (VELAS): an international multicenter observational study on genitourinary syndrome of menopause and stress urinary incontinence. Climacteric. 2015; 18 Suppl 1: 43-48, doi: 10.3109/13697137.2015.1071608, indexed in Pubmed: 26366800. 
54. Pitsouni E, Grigoriadis T, Tsiveleka A, et al. Microablative fractional CO 2 -laser therapy and the genitourinary syndrome of menopause: An observational study. Maturitas. 2016; 94: 131-136, doi: 10.1016/j. maturitas.2016.09.012

55. Siliquini GP, Tuninetti V, Bounous VE, et al. Fractional CO2 laser therapy: a new challenge for vulvovaginal atrophy in postmenopausal women. Climacteric. 2017; 20(4):379-384, doi: 10.1080/13697137.2017.1319815, indexed in Pubmed: 28503946.

56. Behnia-Willison F, Sarraf S, Miller J, et al. Safety and long-term efficacy of fractional $\mathrm{CO} 2$ laser treatment in women suffering from genitourinary syndrome of menopause. Eur J Obstet Gynecol Reprod Biol. 2017; 213 39-44, doi: 10.1016/j.ejogrb.2017.03.036, indexed in Pubmed: 28419911.

57. Zerbinati $N$, Serati M, Origoni M, et al. Microscopic and ultrastructural modifications of postmenopausal atrophic vaginal mucosa after fractional carbon dioxide laser treatment. Lasers Med Sci. 2015; 30(1): 429-436, doi: 10.1007/s10103-014-1677-2, indexed in Pubmed: 25410301.

58. Pitsouni E, Grigoriadis T, Falagas ME, et al. Laser therapy for the genitourinary syndrome of menopause. A systematic review and meta-analysis. Maturitas. 2017; 103: 78-88, doi: 10.1016/j.maturitas.2017.06.029, indexed in Pubmed: 28778337. 